\title{
Monitoring of Cleanliness Level in Hydraulic Systems; Obtaining Reliable On-Line data
}

\author{
홍정희 · 마이크 데이 \\ Jeong-Hee Hong and Mike Day
}

Summary: Monitoring of system cleanliness levels and counting of particulate contaminant are fundamental to achieving hydraulic system reliability as any departure from the specified cleanliness level is often a precursor to future failures. On-line monitoring of cleanliness levels has the advantage of giving data both very quickly and accurately as environmental influences are eliminated. In this way, corrective actions can be promptly implemented. Most on-line instruments are sensitive to system conditions to a greater or lesser extent, but Automatic Particle Counters (APCs) working on light extinction principles are especially sensitive to the presence of optical interfaces caused by such conditions as fluid mixtures, emulsions, free water and air bubbles. These conditions give erroneous data and can result in drawing incorrect conclusions, wasting maintenance time and ultimately, reduced user confidence in cleanliness monitoring. This paper describes such conditions and shows how the correct selection of the analysis technique can result in reliable cleanliness level data.

\section{1. 서 론}

In today's economic climate, companies are striving to achieve improvements in efficiency in order to enhance profitability, and each area of the operation is being critically examined. Improvements in product quality and production or process efficiency are the two keystones for increased profitability. One such area is in the reduction of so called tribological failures in hydraulic equipment. A paper by Day states that the costs of such failures are substantial [1] and also is readily reduced with current technology. The presence of solid particulate contamination in the hydraulic fluid has been found to be the major cause of failures in these systems [2, 3] as it can cause a variety of problems from catastrophic failure by jamming components through to reduced component service life by wear.

The extent of solid contamination has a direct bearing on the performance and reliability of the system and it is necessary that it is controlled to levels that are considered appropriate for both the contaminant sensitivity of the system concerned and the life and reliability required by the operator.

Operators of hydraulic equipment are gradually defining maximum particle concentration levels for components, system and processes, beyond which corrective actions are implemented to normalize the levels. These are often referred to as the Required Cleanliness Level (RCL). The cleanliness level of a process is obtained by sampling the hydraulic fluid and measuring the level of particulate contamination. If the level is above the RCL, then corrective actions are necessary to restore the situation. To avoid taking unnecessary actions, which can often prove costly, precision in sampling and measuring the particulate contamination level is required.

The paper looks at the options available to the

\footnotetext{
Jeong-hee Hong: Pall Korea Limited, SLS

E-mail: Jeong-hee_Hong@Pall.com

ISO/TC131/SC6 \& ISO/TC20/SC10 KATS

Mike Day: Consultant, Pall Europe Limited,

E-mail: Mike Day mikejday31@gmail.com

ISO/TC131/SC6 \& ISO/TC20/SC10 BSI
} 
user, examines the potential accuracy of candidate techniques and looks at the influence of system conditions on the data. It shows how the correct selection of analytical technique and application of proper procedures can overcome these errors.

\section{Philosophy of Fluid Cleanliness Monitoring}

To achieve the potential savings stated above, the fluid cleanliness control measures of the system or process (not just the filter) must be operating at optimum efficiency and should be monitored on a continuous basis. Any small reduction in the fluid cleanliness level can then be promptly identified and immediately rectified. This way, the wear to the system and hence the amount of material removed from the components surfaces is minimized.

To appreciate why this is necessary one has to have a basic understanding of the wear processes involved. If the filter has been correctly selected, it is properly maintained and the lubricant properties are within specification, then the fluid system will operate at its design value. In this situation the particles generated by the system will be low numbers of small particles, generally less than $15 \mu \mathrm{m}$ (Figure 1).

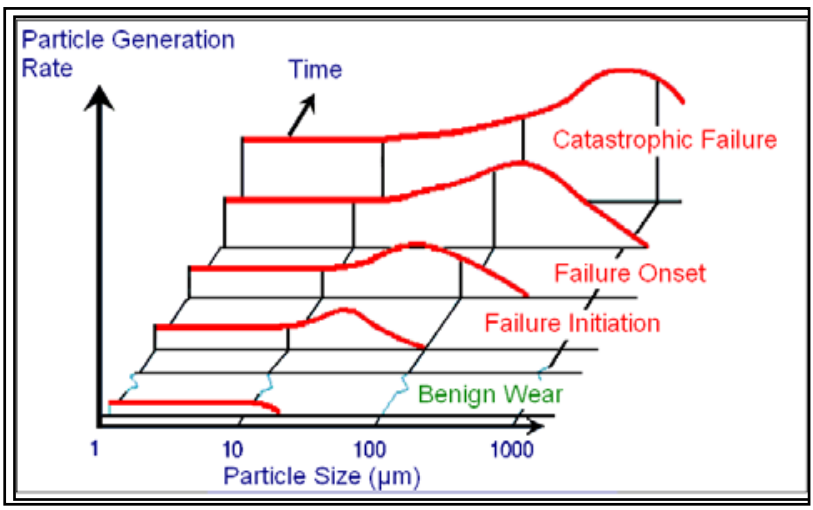

Fig. 1 Particle generation rate of components leading up to failure.

These particles are produced by changing stress at the surface of the component such that the surface fails and particles are released. They are termed "fatigue platlets". This is a very mild form of wear (called "benign wear") and the particles produced can readily pass through the dynamic clearances and will only produce a minimal amount of wear by three-body interaction.

If there are system changes, such as the incorrect grade of filter is fitted, the performance of the filter degrades in service, or the lubricity of the hydraulic fluid decreases, then the balance is disturbed and wear rates will increase. Unless this increase is identified and rectified, regenerative wear will result; both the numbers and the size of the particles generated will increase rapidly. The wear regime will change from mild fatigue wear through to severe abrasive wear, where substantial amounts of surface material are removed. Component life is thereby immediately reduced, the system becomes unreliable and failure, perhaps catastrophic in nature, may be experienced. In this regenerative wear situation, the time between an "out of specification" condition and a failure is relatively short. Even if a failure is not experienced, the removal of surface material will reduce the components' service life. Thus, all the good work done earlier by implementing cleanliness control measures has been wasted. The result is a substantial increase operating costs as unplanned stops reduce production and output, increase maintenance costs and have a knock-on effect on other schedules.

Modern maintenance regimes, whether they are called Predictive Maintenance or Reliability Centered Maintenance, to name two, all feature the measurement of a performance parameter and corrective actions are taken when the measured level exceeds stated limits. In view of the importance of fluid cleanliness to the life and reliability of the system, Cleanliness Monitoring should be taken on as a front line technique and applied with other forms of monitoring. The paper by Hunt [4] reiterates this and states that of all of the techniques available for monitoring hydraulic systems, cleanliness monitoring provides the earliest and most cost effective warning of potential problems,

\section{Options for Particle Monitoring}

\section{3-1. Modes of Operation}

There are three basic modes of operation, as shown 
in Figure 2. :

- off-line from a sample bottle or container;

- on-line from a operational flow line;

- in-line from a pressurized line

\section{a) Off-line}

Off-line analysis involves the extraction of a representative sample of the system fluid, and its collection in either a sample bottle or suitable container for subsequent analysis. Analysis can be either adjacent to the process, at an in-house laboratory or sent away to an external laboratory. If external laboratories are used, it can greatly extend the period between sampling and implementing corrective actions.

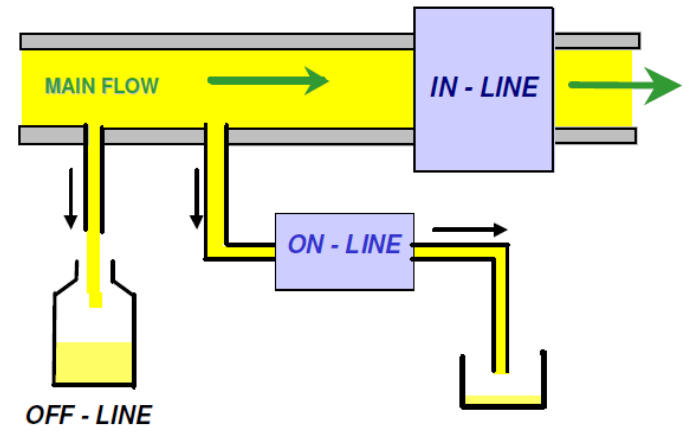

Fig. 2. Schematic diagram of modes of operation

The process of sampling and subsequent preparation for analysis will add extraneous contamination into the sample from the environment or the process itself. This will introduce errors and variability in the data. Thus it is essential that approved procedures are used to limit such errors. For samples from modern hydraulic systems, this extra dirt can greatly inflate the contamination level to such an extent that the true cleanliness level cannot be determined. This will effectively put a limit on the minimum cleanliness level that can be reported using this method. The subject has been researched by Tampere University of Technology (TUT) [5] who compared duplicate on-line and off-line bottle samples from 133 systems. The results are summarized in Figure 3 where the error caused by bottle analysis is plotted against the 'true' value as represented by the on-line data. The contamination level is represented by the ISO 4406 Solid Contamination Coding system [6] where the interval between each code is effectively a doubling of the contamination level. The graph shows how, as the system levels get cleaner, the error in data increases. The TUT data indicated that the limit for bottle sampling is about ISO 16/14/12 and even at this level there will be an error of about 50\%. Thus, there could be a substantial increase in the contamination level and it may go unnoticed. For example, a system is operating at ISO 8 at $5 \mu \mathrm{m}$ by on-line analysis, but if a bottle sample was analyzed there could be a +5 code increase through errors as seen in Figure 3, giving a ISO 4406 code at $5 \mu \mathrm{m}$ of ISO 13. Now if the generation rate increased to 12 (a 16 fold increase) then the bottle analysis would record $12+$ an error of $2=$ ISO 14 . This change would not cause concern to users of bottle analysis!

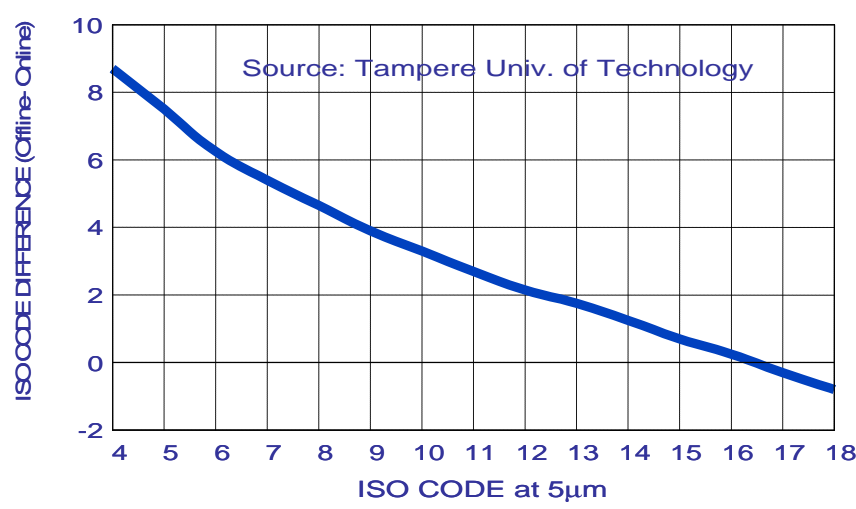

Fig. 3. Comparison of on-line and sample bottle data

\section{b) On-line}

In on-line analysis the instrument is connected either directly to the pipework that sees active flow or to a subsidiary flow line taken from it. This method of analysis overcomes the problems associated with the addition of environmental contamination seen above and can give the 'true' result, There are two ways of applying this technique; using a portable analysis unit which is connected to a number of systems in turn perhaps on a maintenance route, or by having a unit permanently installed.

With a portable unit, contamination can be generated, when the unit is connected to the system 
and unless this is flushed out before analysis, errors will result. Most of the on-line instruments only use a small flow rate (20 to $100 \mathrm{~mL} / \mathrm{min}$ ) and this is often insufficient to flush the lines after connection. Hence, either long flushing times are necessary or the pipes have to be pre-flushing before connection.

Permanently connected units do not have this limitation.

\section{c) In-Line Analysis}

Instruments working in-line are permanently connected to a flow line and, hence, provide continuous measurement thereby overcoming the need of flushing. Unfortunately there are now no instruments of this type available to the user at present.

\subsection{Options For On- Line Analysis}

Here are really only two options for on-line analysis the Automatic Particle Counters or monitors (hereafter collectively labeled as APCs) and the Filter (or Mesh Blockage Instruments (hereafter called FBMs). These are briefly described below and further described in a paper by Day \& Hong [7].

\section{a) Automatic Particle Counters}

These instruments work on the light extinction principle where a light beam illuminates a narrow passage way through which the contaminated fluid flows. Particles in the fluid cause obscuration (blockage or scattering) of the light beam in proportion to their size and produce corresponding electrical signals. The number and magnitude of signals is used to determine the particle size distribution. The relationship between particle size and amount of light blocked is obtained by calibration [8]

Without the development of APCs, much of the research into contamination and filtration in hydraulic systems over the last 40 years would not have been possible. When used within their limitations, APCs have demonstrated both accuracy and economy of operation. The APC can cater for a wide particle size range, from 0.5 to over $2000 \mu \mathrm{m}$ depending on the type of instrument and its application, and they can work directly in-line, on-line in the 'sip' or suction mode from low pressure sources or from bottle samples extracted from the system. However, they are subject to certain limitations and, if not used correctly, can give erroneous counts and this is discussed below.

There are two main problem areas; optical interfaces in the fluid created by the presence of other fluids such as air bubbles, water droplets, liquid additive droplets (typically silicone anti-foam additive), immiscible liquids such as oils and greases in water glycol fluids, etc. All of these interferences will cause a reduction in light received by the detector and will be recorded erroneously as particles. The other major source of error is due to "coincidence" i.e. the presence of more than one particle in the sensor at the same time. Although this will usually occur when the concentration of particles is too high, it will also be experienced when there is large amounts of small particles (say $<3 \mu \mathrm{m}$ ) that are generally smaller than the minimum size.. Counted together, they combine to produce a single larger particle and so inflate the numbers of particles close to the minimum size. Such particles are called "silt" and can be generated by chemical reactions with the fluid producing a precipitate or they can be particles produced by either the system (gearboxes are notorious for this!) or by the filter which cannot or does not hold on to these particles. Examples of these effects are given in section 4 .

\section{b) Filter or Mesh Blockage Monitors (FBM)}

These instruments were designed to provide an on-line and an off-line solution where the condition of the fluid precludes the use of an APC as described above. The principle of FBMs is that the blockage of a filter or disc with regular sized openings or pores is a function of the number of particles in the fluid whose size is greater than the filter's pore size. The capture of these particles will induce partial blockage of the filter and increase the differential pressure across it in proportion to the numbers of particles in the fluid. After the analysis cycle, the filter is cleaned by reversing the flow and 
back flushing the particles off the filter in readiness for the next cycle.

The differential pressure and viscosity are measured at the start and end of the analysis cycle and the Cleanliness class at the measured sizes is computed after any correction for changes in viscosity. The relationship between Cleanliness level and degree of blockage of the filter, is obtained by calibration as defined in ISO 21018-3 [9], and this unit presents the cleanliness level as codes at $>6$ and $>14 \mu \mathrm{m}$.

Generally this technique is not affected by most of the fluid conditions that affect APCs i.e. water in oil (and vice versa), air bubbles, fluid mixtures and "silts" provided that the state or the mixture remains reasonably constant over the analysis cycle (about 1 minute) so that a valid correction can be made for viscosity changes.

\section{Pit-Falls in Monitoring}

\section{4-1. General Levels of Accuracy}

A fundamental part of any condition based monitoring program is the existence of both a specification for the particular parameter being monitored and documented actions that have to be implemented if there is a departure from the specified performance. Therefore, it is essential that the output data from the instrument is both accurate and consistent otherwise maintenance of the process or system will be initiated, only to find that the out of control situation was a false alarm because the data was in error. Hence, time and effort would be wasted. If this continues then confidence in the instrument will be lost and perhaps cleanliness monitoring will be abandoned. If this happens, these operators will not realize the benefits to be gained from cleanliness monitoring.

Thus accuracy is an important selection parameter and it is based upon two criteria, repeatability which can be said to be the accuracy of the same instrument type to record identical samples, and reproducibility which is the accuracy shown by the same instrument type to record identical samples at different locations. Both types of accuracy are dependent upon the numbers of particles counted, with variability increasing with reducing particle numbers. Unfortunately this is often not appreciated by the user. A study by Day [5] has evaluated the accuracy of various techniques working both on and off-line and this is summarized in Table1. The APC and FBM instruments analyzed prepared fluid samples of a containing a classified test dust both on and off-line, whereas the microscope data was obtained from the analysis of 'real' fluid samples but in one laboratory using three operators. Also, none of these instruments were affected by any of the conditions described in section 4

This data can be considered to be the best possible as it was obtained in the laboratory under controlled conditions, with skilled operators and often with a classified contaminant. Of all of the techniques the APC is the most accurate. The Coefficient of Variance (COV) of the APC and FBM is seen to increase with size and this reflects the statistical effects associated with reducing particle numbers as the size increases. Interestingly, the microscope is not so affected by this phenomenon as the counting method dictates the counting of a minimum number of particles so that the statistics are enhanced. Generally, the variations in reproducibility are usually greater than those of repeatability as other factors come into effect.

Table 1 Accuracy levels of various monitoring techniques

\begin{tabular}{|c|c|c|c|c|}
\hline & Coeffic & th of Vari & ice $(\sigma / x \%$ & t size \\
\hline Technique/Mode & $>5 \mu \mathrm{m}$ & $>15 \mu \mathrm{m}$ & $>25 \mu \mathrm{m}$ & $>50 \mu \mathrm{m}$ \\
\hline Repeatability & & & & \\
\hline APCs - On-Line ${ }^{1}$ & $1.9 \%$ & $3.0 \%$ & $3.9 \%$ & $9.5 \%$ \\
\hline APCs - Bottles ${ }^{1}$ & $2.5 \%$ & $3.7 \%$ & $7.5 \%$ & $20.1 \%$ \\
\hline $\begin{array}{l}\text { Filter blockage monitor } \\
\text { On-Line }{ }^{2,3}\end{array}$ & $3.2 \%$ & $5.9 \%$ & - & - \\
\hline Microscope-Bottles & $8.3 \%$ & $10.4 \%$ & $14.3 \%$ & $10.2 \%$ \\
\hline Reproducibility & & & & \\
\hline APCs on-line ${ }^{1}$ & $5.1 \%$ & $10.3 \%$ & $13.1 \%$ & $32.6 \%$ \\
\hline $\begin{array}{l}\text { Filter blockage monitor - } \\
\text { On-Line }\end{array}$ & $4.2 \%$ & $3.2 \%$ & - & - \\
\hline $\begin{array}{l}\text { Microscope-Bottles } \\
\text { (same laboratory, different } \\
\text { operators) }\end{array}$ & $14.2 \%$ & $13.8 \%$ & $15.6 \%$ & $28.2 \%$ \\
\hline
\end{tabular}

Notes:

1-sizes for APCs relate to ISO 4402 calibration;

2- Sizes relate to earlier 'field' calibration;

3- Presented to ISO/TC131/SC6/WG1 Document N97 
The advantage of operating on-line can be clearly seen from the above data as this mode of analysis is devoid of errors due to sampling, handling or analysis

\section{4-2. Effects of Water Content In Oil on On-Line Particulate Contamination Data}

The effects that water in oil has on data can take two forms. It can directly interfere with the technique i.e. the water droplets affecting light beam or measurement principle, or it can be indirectly interfere by generating a silt layer which may cause coincidence errors (see also 4.4). Both of these instances are present in the next example. The data was obtained from a trial using a vacuum dehydrator (purifier) to remove the water from synthetic oil used in the offshore industry. This provided the opportunity to study the effect of the water on both an APC and FBM operating on-line, as the concentration of water gradually reduced. The vacuum purifier removes free water, dissolved water down to $20 \% \mathrm{RH}$, dissolved gases and also particles. A $7 \mu \mathrm{m}$ (c) ${ }^{1)}$ filter was used in the purifier for the trial and very clean fluid levels were achieved. The results are seen in Figure 4. The output of the FBM was converted into particle counts rather that the usual ISO 4406 codes and the particle count data of both was converted into decimal ISO 4406 codes for better resolution. In addition, a water sensor was used to measure the dissolved water content (black broken line) and this instrument only measures dissolved water i.e. $<100 \%$ RH.

Both the APC and FBM recorded clean particulate contamination levels before the addition of 3000 PPM of water. Note that saturation or $100 \% \mathrm{RH}$ value for this oil is about 700 PPM at the test temperature of $34^{\circ} \mathrm{C}$. After mixing and the creation of an emulsion, the effect of water on the APC can be seen. For a period of time the same ISO codes were recorded at the three ISO sizes of $>4,>6$ and $>14$ $\mu \mathrm{m}$ (c) e.g. ISO 22/22/22. At this stage the water has been broken up into millions of minute water droplets by shearing in the system and passage through the filter and this caused saturation of the APC electronics. The APC data is substantially affected by both coincidence (droplets combining to produce bigger' particles') and saturation (missing particles). The data from the FBM, on the other hand, is hardly affected by the condition of the oil and gives realistic and consistent data throughout the test. A small increase in code is seen in the early stages when conditions are unstable as the emulsion is created.

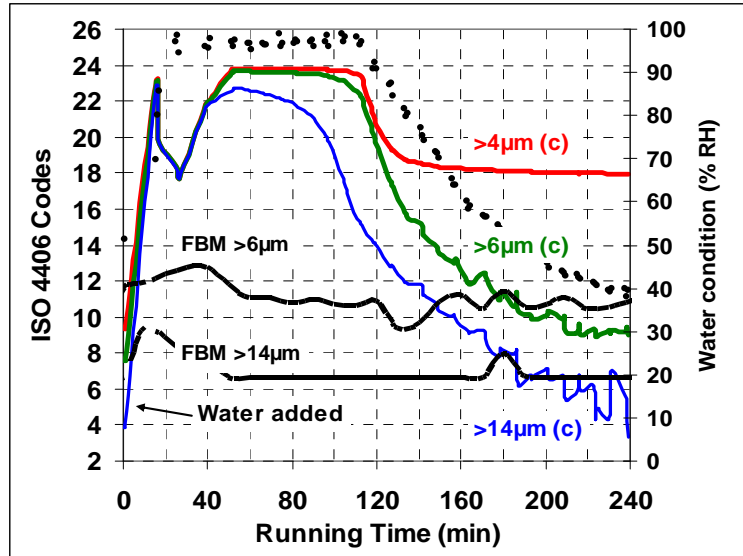

Fig. 4. Variation of ISO 4406 codes of APC and FBM during water variance

After about 120 minutes the data from the Water Sensor (broken black line) showed that the water was below saturation level $(<100 \% \mathrm{RH})$ and was in a "dissolved" state. However, there was still a significant difference between the $>6 \mu \mathrm{m}$ and $>14 \mu \mathrm{m}$ data recorded by the FBM and the APC, and it is not until after another 120 minutes that the data agreed for the $>6 \mu \mathrm{m}$ particles. Note that the APC data at $>4 \mu \mathrm{m}$ shows an unexpectedly slow rate of reduction as the high efficiency $7 \mu \mathrm{m}$ filter should have reduced $>4 \mu \mathrm{m}$ particles much more quickly than seen in Figure 4. It is therefore concluded that the particles are much smaller than $4 \mu \mathrm{m}(\mathrm{c})$. It is thought that these very small particles were caused by a reaction between the oil additives and the water and are increasing the numbers of $4 \mu \mathrm{m}(\mathrm{c})$ particles by coincidence. However the presence of water micro-droplets creating this effect cannot be discounted and this phenomenon is further described in section 4.4. 


\section{4-4 Effects of Dissolved Water Content In Oil} On Off-Line Particulate Contamination Data

To investigate what happens to the APC at the transition between dissolved and free water phases, a study was conducted by Pall Corporation and the results are summarised here.

A series of bottle samples were generated with oil at various dissolved water content levels (20, 30, 40, $50,60,70,80,90 \& 95 \%$ of saturation or $\mathrm{RH}$ ) at the same temperature. The oil used was one that has better tolerance to water than the one used in 4.3. The bottle samples were analysed by an APC and a FBM. The results are shown in Fig.6

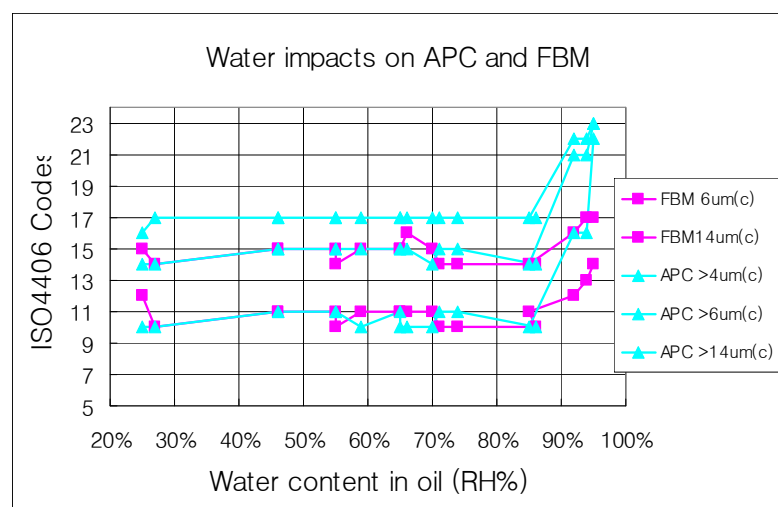

Fig. 5. Variation of ISO 4406 codes of APC and FBM in Dissolved water content tests

In the range of $25 \% \mathrm{RH}$ to $85 \% \mathrm{RH}$, the increased water content did not affect the data and 'reasonable' agreement between the APC and FBM is seen. However, when the water content was over 85\% greatly inflated particle counts were recorded by the APC compared to the FBM. The contamination levels recorded by the APC at $6 \mu \mathrm{m}$ (c) were about 5 ISO codes or 32 times higher than the FBM.

Note: The data has to be viewed comparatively as there was an increase in the contamination level of later samples due to the use of high dilution ratios which increased the particle counts recorded by both the FBM and APC.

\section{4-5 Effects of Air Bubbles on Particulate Contamination Data}

Air in liquid systems will be problematic to APCs only if it is entrained or comes out of solution. It will then create an optical interface similar to that of water and be recorded as a particle by the APC. This can be endemic in both low pressure lube applications and in gear and bearing systems. The FBM is not generally affected if the state of the air is uniform and continuous during the analysis cycle.

The effects of air has been simulated by a laboratory test where air was injected into a low pressure $(<4$ bar) recirculatory rig to increase the entrained air content. Monitoring was performed using two APCs situated in different locations, with APC 1 at a higher pressure and an FBM positioned in between the air injection point and the reservoir. This represents a very severe test of the FBM. A number of tests were performed, including the effect of a $5 \mu \mathrm{m}$ (c) filter on the particle counts, and the data is presented in tabular form in Table 2. The data is the average of three APC runs and two FBM runs as the FBM analyses a larger volume. Again the data is presented in terms of decimal ISO 4406 codes.

Table 2 Effect of air on FBM and APC data.

\begin{tabular}{|c|l|c|c|c|c|c|c|c|c|}
\hline \multirow{2}{*}{$\begin{array}{c}\text { Test } \\
\text { Number }\end{array}$} & Descripn & \multicolumn{3}{|c|}{ APC 1 } & \multicolumn{3}{c|}{ APC 2 } & \multicolumn{2}{c|}{ FBM } \\
\cline { 2 - 10 } & $4 \mu \mathrm{m}$ & $6 \mu \mathrm{m}$ & $14 \mu \mathrm{m}$ & $4 \mu \mathrm{m}$ & $6 \mu \mathrm{m}$ & $14 \mu \mathrm{m}$ & $6 \mu \mathrm{m}$ & $14 \mu \mathrm{m}$ \\
\hline 1 & Filters in & 12.1 & 10.1 & 7.2 & 10.2 & 8.1 & $<1$ & 10.2 & 6.7 \\
\hline 2 & $\begin{array}{l}\text { Filters } \\
\text { out }\end{array}$ & 13.8 & 11.5 & 6.5 & 13.7 & 11.1 & $<1$ & 10.6 & 7.7 \\
\hline 3 & $\begin{array}{l}\text { Filters } \\
\text { out, } \\
\text { air on }\end{array}$ & 18.8 & 17.8 & 14.2 & 18.6 & 17.4 & 12.7 & 11.3 & 8.1 \\
\hline 4 & Filters in & 11.6 & 10.1 & 6.1 & 11.7 & 10.1 & 4.3 & 10.8 & 7.0 \\
\hline 5 & $\begin{array}{l}\text { Filters in, } \\
\text { air on }\end{array}$ & 20.8 & 13.2 & 8.8 & 14.7 & 11.7 & 6.4 & 12.9 & 8.6 \\
\hline 6 & Filters in. & 11.8 & 7.4 & 3.8 & 11.7 & 9.0 & $<1$ & 12.3 & 9.6 \\
\hline 7 & $\begin{array}{l}\text { Filters } \\
\text { out, } \\
\text { air on }\end{array}$ & 19.9 & 12.9 & 11.9 & 18.9 & 18.6 & 18.0 & 13.2 & 9.3 \\
\hline
\end{tabular}

Overall the APCs were always affected by the presence of air to varying degrees and their operation became unpredictable. On the other hand, the FBM was mostly unaffected despite the severity of the tests. The amount of air that was injected was quite substantial and caused frothing in the reservoir. The magnitude of the effect on the APC depended on a number of factors, e.g. the sampling position around the system and static pressure (APC1 \& APC2), distance from air source, connection pipe length and 
whether the filter was in or not (tests $3 \& 5$ ). The errors were characterized by higher ISO codes and, and often near identical codes at adjacent sizes where air was substantial (Tests 3 and 7-APC2). When the filter was in circuit the bubble size was reduced and large code differences between adjacent codes were seen (Tests 5 \& 7).

\section{4-6 Effects of Small Particles in Gear Oil in Tractor Trans-Axles}

The error seen in this example is perhaps the major cause of errors in particle count data and is prevalent amongst most if not all particle counting techniques. It can be generated by a wide range of particle types (and some are not 'real' particles); examples are: small fatigue and rubbing wear particles which are nearly two dimensional (i.e. very thin); oil additives e.g. silicone anti-foam additives, extreme pressure additives and molybdenum disulphide anti-wear additive; chemical reaction products and carbon caused by 'dieseling' of the oil. All of these particles are small $(<3 \mu \mathrm{m})$, are present in large numbers and give coincidence errors in one form or another.

With the APC, 'many' particles will be present in the sensor at the same time and although their size may be below the APC's minimum size they will be detected and will be sized as a single particle of a larger size. The error is characterized by large code differences between the $2^{\text {nd }}$ and $3^{\text {rd }}$ ISO 4406 codes e.g. ISO 20/18/11, whereas a difference of 3-4 codes is usual. The above 'silt' examples rarely cause problems with the FBM as the sizes of the particles are usually well below the rating of the finest filter or mesh and the particles pass through. If the particle size is sufficient to be captured by the smallest mesh it is no longer considered to be "silt".

Microscope counting can also be affected by high populations of small particles and if particles are overlapping they can be oversized. The result is an overcount of larger sizes and an undercount of smaller sizes. Happily, this high concentration will be seen when viewing the membrane filter prior to counting and, in this case, the membrane filter should be rejected and a new one prepared using a smaller sample volume.

The mechanism of coincidence in APCs is best illustrated from laboratory tests performed on a sample taken during flushing of an agricultural tractor trans-axle which contains the gearbox, clutch and sometimes brakes. The operator used an APC to verify the "Roll-off" cleanliness. He complained that the $6 \mu \mathrm{m}$ filter was not working effectively and the contamination level recorded after 50 tractors had been processed was ISO 20/19/11. This exceeded the specification of ISO -/17/14. A microscope count gave $-/ 14 / 12$ but revealed a high level of small particles $(<3 \mu \mathrm{m})$ or "silt". Coincidence effects with the customers APC were suspected.

Table 3 Analysis of samples from a tractor trans-axle

\begin{tabular}{|c|c|rr|r|r|}
\hline \multirow{2}{*}{$\begin{array}{c}\text { DILUTION } \\
\text { FACTOR }\end{array}$} & \multicolumn{4}{|c|}{ PARTICLE COUNTS PER 100mL } & \multirow{2}{*}{ ISO CODES } \\
\cline { 2 - 5 } & $>2 \mu \mathrm{m}$ & $>5 \mu \mathrm{m}$ & $>15 \mu \mathrm{m}$ & $>25 \mu \mathrm{m}$ & $2 / 5 / 15 \mu \mathrm{m}$ \\
\hline MICROSCOPE & - & 16,200 & 1,320 & 324 & $* / 14 / 11$ \\
\hline FBM & \multicolumn{4}{|c|}{ Data output is only as ISO 4406 Codes } & $-/ 15 / 12$ \\
\hline APC & $1,710,000$ & $1,020,000$ & 1,410 & 352 & $21 / 21 / 11$ \\
\hline X4 & $4,760,000$ & 406,000 & 1,100 & 304 & $23 / 19 / 11$ \\
X8 & $5,820,000$ & 49,400 & 992 & 224 & $23 / 16 / 10$ \\
X16 & $2,930,000$ & 20,700 & 1,090 & 320 & $22 / 15 / 11$ \\
X32 & $1,510,000$ & 18,600 & 1,020 & 256 & $21 / 15 / 11$ \\
X64 & 669,400 & 18,400 & 3,580 & 256 & $20 / 15 / 12$ \\
\hline
\end{tabular}

A larger sample was obtained from a tractor under test and analyzed using a microscope, an APC but with different dilution ratios and an FBM. The data is presented in Table 3. The microscope count confirmed the consistency of the samples and a similar result was obtained from the FBM. It is when the APC data is examined that the reason for the original discrepancy is seen.

The cause of the initial error is both coincidence and saturation. In the undiluted sample the numbers of small particles are so high that the APC electronics does not reset correctly and undercounts the $>2 \mu \mathrm{m}$ particles due to saturation and over counts the $>5 \mu \mathrm{m}$ due to the coincident particles. As the dilution ratio increases the counts at $>2 \mu \mathrm{m}$ firstly increase as saturation effects are reduced and then 
both the $>2 \mu \mathrm{m}$ and $>5 \mu \mathrm{m}$ counts decrease as coincidence errors are then reduced. However, it was not until high dilution ratios were used ( $>$ x64) that a reasonable representation was obtained.

It should be stated that coincidence errors are rarely experienced with clean samples say, ISO $17 / 15 / 12$, provided that it does not contain silt from the oil decomposition process.

Also it must be remembered that with on-line APC analysis the samples cannot be diluted if a problem is suspected and the results have to be believed, A good check is to look at the difference in the ISO codes given by an APC at the 6 and $14 \mu \mathrm{m}$ (c) level. If it is $>4$ codes then coincidence .errors should be suspected.. If these are confirmed then a sample will have to be taken for laboratory analysis including dilution.

\section{4-7 Effects Of Anti-Foam Additives On APC Counting Accuracy For ATF Oil}

Some anti-foam additives notably silicone anti-foam (SAFA) additive are not soluble in oils and exist as minute droplets, generally $<2 \mu \mathrm{m}$. Although the concentration of SAFA is generally small (about 5 PPMv) this represents a huge number of $2 \mu \mathrm{m}$ droplets; approximately 450 million per $100 \mathrm{~mL}$ ! If these are detected by the APC errors will be generated through the mechanism of coincidence in a similar manner to the example in 4.6.

The effect of this is illustrated by the following example. A manufacturer of automotive Direct Shift Gearboxes (DSG) used Image Analysis (IA techniques) to analyze samples taken from a DSG test facility. Although, satisfactory results were obtained, the operator was not satisfied with the 1 to 2 hours needed to process a sample. As on-line APC analysis offered the prospect of greatly reducing processing time, Pall assisted the OEM in setting up an APC to measure the cleanliness of the oil. As these gear boxes generate air bubbles in the oil, a high pressure pump and loading valve were used to increase the pressure in the APC sensor to increase the oil's solubility and forces any air into solution. Although the system was capable of pressures up to 100 bar, 50 bar was used successfully for these tests, The arrangement was installed at the ATF Oil Storage System (OSS) downstream of $6 \mu \mathrm{m}$ grade filters.

The results of the APC (on-line) and IA analysis are seen in Figure 6 and the particle counts are represented by the ISO 4406 cleanliness codes with the IA data presented at $>2 \mu \mathrm{m},>5 \mu \mathrm{m},>15 \mu \mathrm{m}$ and the APC at $>4 \mu \mathrm{m}$ (c), $>6 \mu \mathrm{m}$ (c), $>14 \mu \mathrm{m}$ (c). To get a better comparison between the APC and IA, fluid samples were sampled as simultaneous as possible.

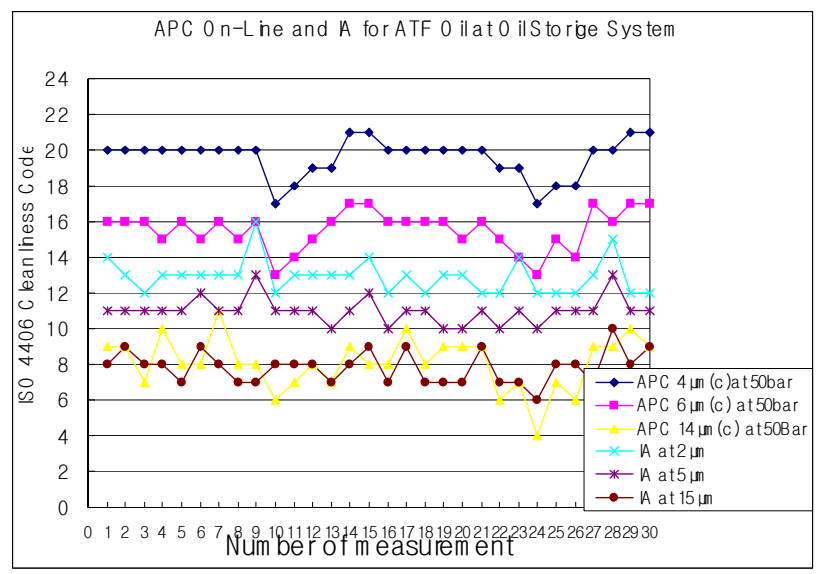

Fig 6. Test Results from Oil Storage System (OSS)

It must be appreciated that the results of the APC and IA are not directly comparable as both the measurement parameters and analysis modes are different. Accepting this, when the data is compared there are substantial differences between the APC and IA data at the particle sizes $<10 \mu \mathrm{m}$. The IA data would be expected to be higher than the APC data because of the use of sample bottles (see section 3.1), but instead the APC data is substantially higher for similar sizes $(2 / 4 \mu \mathrm{m}(\mathrm{c})$ and $5 / 6 \mu \mathrm{m}$ (c) by up to 7 and 3 ISO codes respectively at the two smallest sizes, This is about 128 and 8 times higher!!)

As the pressurization to 50 bar eliminated air bubble effects, the only reason left for the higher ISO codes was that the APC was counting the anti-foam additive.

\section{4-8 Problems Associated With Monitoring Cleanliness Levels in Water Glycol (WG) Fluids} Water glycol (WG) hydraulic fluids are usually a 
solution of glycol in water with additives added for specific applications. In this 'pure' state they should not give rise to particle counting problems. However if WGs are contaminated with even traces of oils, greases or any other immiscible liquids then substantial errors can be experienced with APCs, Immiscible droplets are generated by the system (shearing, passage through filters etc) and they interfere with the passage of light, much in the way that SAFA did. Using the same approach as earlier 1PPMv could generate 12 million droplets sized $4 \mu \mathrm{m}(\mathrm{c})$ or an ISO code of 24 !

In addition to this problem, there are a family of Sub-sea control fluids that are micro-emulsions which can give rise to further counting problems, Although the emulsion droplet size is small $(<2 \mu \mathrm{m})$ and should be well below the lower threshold of an APC, they will be detected as 'noise' and produce erroneous results.

The problems associated with APC counting WG fluids are illustrated by the following example. A hydraulic flushing skid used for flushing Off-shore systems was used to flush the WG fluid in a thrust hydraulic system that is mounted on an offshore drill ship to control the ship's balance. The pump was rated at $1500 \mathrm{~L} / \mathrm{min}$ and it was fitted with large filters rated at $\beta>1000$ at $5 \mu \mathrm{m}$ (c).

During flushing very high particle counts were measured when an APC was used to monitor the cleanliness of the fluid. As these were unrepresentative of the grade of filter used, the contamination level was further investigated with an on-line FBM and an off-line IA instrument which analyzed sample bottles containing fluid extracted from the system. The results of two analysis taken towards the end of the flushing program are seen in Table 5. The errors that are generated by the oil contamination on the WG fluid can clearly be seen. This is typical of extreme coincidence that causes the APC to saturate and give very similar ISO codes across the sizes.

The FBM was not affected by this contamination and its output was confirmed by the IA data.
Table 5 Comparison test samples between APC and FBM and OPC for WG Oil samples

\begin{tabular}{|cccc|}
\hline SAMPLE & FBM & APC & OPC \\
\hline & On-line & On-line & \\
$\# 1$, & ISO 4406 & ISO & ISO-/15/11 \\
\hline & $-/ 14 / 10$ & $22 / 21 / 20$ & \\
$\# 2$, & ISO 4406 & ISO & ISO-/15/11 \\
\hline
\end{tabular}

\section{Conclusions}

Monitoring the cleanliness of a hydraulic fluid as part of a Predictive Maintenance regime is acknowledged to be the most sensitive monitoring technique as it can detect adverse changes before they becomes an issue and long before a problem is experienced.

For cleanliness monitoring to be most effective any potential change has to be very quickly detected and promptly corrected. On-line monitoring is the way to achieve this.

The on-line technique chosen has to give accurate and reliable data, otherwise a lot of time and effort could be wasted by acting on incorrect data.

Automatic Particle Counters (APCs) have proved indispensable in furthering contamination studies because of their high accuracy and speed of operation, when operated under controlled conditions and within their limitations. There are however certain operating conditions that will give rise to monitoring errors. Users must be aware of these conditions and be knowledgeable of them so that they can be realized and the erroneous data ignored.

An important finding in this paper is the effect that dissolved water can have on the APC data when the water content is close to the $100 \% \mathrm{RH}$ or saturation level i.e. $>85 \%$ of RH. Error in data was experienced even though the oil was clear. It is thought that this is because the water is present as micro-bubbles which do not appreciably affect the visual appearance but will be detected by the APC.

The Filter Blockage monitor is insensitive to most of the conditions that affect APCs and provide an alternative to APCs where general monitoring is required. 


\section{References}

[1] M. J. Day, "Condition Monitoring of hydraulic system” Chapter 10, Handbook of Condition Monitoring, ed BKN Rao, Advanced Technology, Oxford, UK, 1996, ISBN/856172341.

[2] E Rabinowicz, Lecture presented to the American Society of Lubricating Engineers, Bearing Workshop, USA, 1981.

[3] DTI, "Contamination control in fluid power systems, Vol 1 Field Studies,” Department Trade and Industry, N.E.L., Glasgow, UK., February 1984.

[4] T.M. HUNT "Handbook of wear debris analysis and particle detection in liquids" published by Chapman \& Hall, London 1993, ISBN 1-85166-962-0.

[5] M. J. Day \& J. Rinkinen: "Contaminant monitoring of hydraulic systems - the need for reliable data”, Presented at COMADEM 97, Tampere University of Technology, Tampere, Finland, 1997.

[6] ISO 4406: Hydraulic Fluid Power - Fluids Method For Coding Level of Contamination by Solid Particles, International Standards Organisation, Geneva, Switzerland, 1999.

[7] J-h. Hong \& M. J. Day: "Filter Blockage Technique,- Monitoring of the Cleanliness in Hydraulic Systems”, Pp150 Pp159, The Proceedings of Korea Fluid Power System Fall Conference, October 22, 2008, Incheon, Korea

[8] ISO 11171:- "Hydraulic fluid power "Calibration of automatic particle counters for liquids" International Standards Organization, Geneva, Switzerland

[9] ISO 21018-3.: "Hydraulic fluid power Monitoring the level of particulate contamination of the fluid - Part 3: Use of the filter blockage technique", International Standards Organization. Geneva, Switzerland.

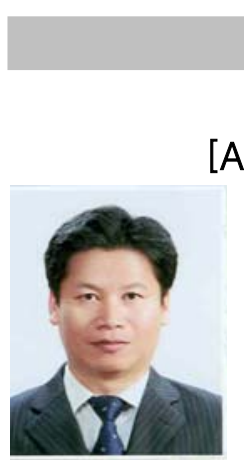

\section{[Authors Introduction]} Jeong-hee Hong(홍정희 理事)

E-Mail:Jeong-hee_Hong@pall.com Tel.:02-560-8791, 010-9070-0669 Born on December 10, 1968. Precision Mechanical Engineering in Busan National University, Bachelor's degree, on Feb. 1991. Precision Mechanical Engineering in Busan National University, Master's degree, on Feb.1993.

Worked at Research \& Development Center in Doosan Infracore; 1993-2000. Now working for Scientific \&Laboratory Service in Pall Korea Limited; 2000 2012. Represents Korea, ISO/TC131/SC6 \& ISO/TC20/SC10 Technical Committee Member in KATS

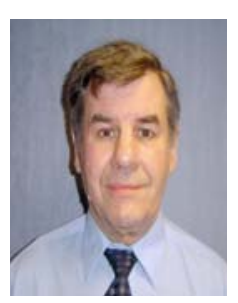

Mike Day

E-Mail: mikejday@gmail.com Tel: +44 (0)2392 251658

Born on July 31, 1946.

Mechanical Engineering at Staffordshire University, Bachelor's degree on July 1970, Cranfield University, Master's degree on July 1984.

Worked for BHR Group Research 1974-1984, worked for Pall Europe Ltd., Scientific \& Laboratory Serviced, 1984- 2005 and Technology Group 2005-2011, Now managing director CMS Consultants Ltd.

Represents UK on ISO/TC131/SC6 and ISO/TC20/SC10. Convener of ISO/TC131/SC6/WG1. 